\title{
Search for low mass Higgs-boson like resonances at CMS
}

\section{Benoit Courbon*t}

Institut de Physique Nucleaire, IN2P3-CNRS

E-mail: benoit.courbon@cern.ch

\begin{abstract}
A search is performed on the $8 \mathrm{TeV}$ LHC data for additional scalars and pseudoscalar with masses below the newly discovered higgs boson $\mathrm{h}(125)$. These searches are motivated within several BSM theories, most significantly extensions of the non mininal extensions of the MSSM like the NMSSM, where additional scalar and pseudoscalar states are expected. The mass range from 250 $\mathrm{MeV}$ to $110 \mathrm{GeV}$ is explored with different final states. The current status of these searches will be reviewed and prospects will be given to extend these searches in the Run2 of the LHC.
\end{abstract}

XXIV International Workshop on Deep-Inelastic Scattering and Related Subjects

11-15 April, 2016

DESY Hamburg, Germany

\footnotetext{
${ }^{*}$ Speaker.

$\dagger$ on behalf of the CMS collaboration
} 


\section{Introduction}

The discovery of a Higgs boson with a mass close to $125 \mathrm{GeV}$ has been a major milestone in the understanding of the origin of the elementary particle masses [1] [2] [3]. All the properties of this boson appear to be in accord with the Standard Model (SM) at the current level of precision [4] [5]. However, the SM cannot address several crucial issues such as the hierarchy problem or the nature of dark matter [6]. Theories beyond the Standard Model (BSM) have been proposed to address these questions. Many of them predict an extended Higgs sector, possibly with an additional low-mass (below $125 \mathrm{GeV}$ ) scalar or pseudoscalar.

Two-Higgs doublet models (2HDM) are a simple extension of the SM [7]. They feature two doublet fields $\phi_{1}$ and $\phi_{2}$, giving rise after $S U(2)_{L}$ symmetry breaking to five physical states : two scalar ( $h$ and $\mathrm{H}$, with $m_{h}<m_{H}$ ), one pseudoscalar $(A)$ and two charged $\left(H^{ \pm}\right)$Higgs bosons. There are four distinct types of $2 \mathrm{HDM}$, depending on which doublet the quarks and leptons couple to. The main parameters of the models are the two doublets vacuum expected values ratio $\tan \beta$ and the mixing angle $\alpha$ of the two scalar bosons. In the "alignment limit" [8], one can obtain a SMlike scalar $H$ or $h$ compatible with the discovered Higgs boson $h(125)$ and an additional low-mass scalar $h$ or pseudoscalar $A$.

Supersymmetric models [9] also predict extended Higgs sectors. First, the Minimal Supersymmetric Standard Model (MSSM), which is a type II 2HDM, features 5 Higgs bosons. However, within this model the existence of a low-mass Higgs boson is strongly disfavoured, both theorically and experimentally. The Next-To-Minimal Supersymmetric Standard Model (NMSSM) is a simple extension of the MSSM, addressing the $\mu$ problem of the latest model. It consists in adding a singlet field $S$ to the two doublets, resulting in the existence of seven physical states : three scalar $\left(h_{1}, h_{2}\right.$ and $h_{3}$, with $m_{h_{1}}<m_{h_{2}}<m_{h_{3}}$ ), two pseudoscalar $\left(a_{1}\right.$ and $\left.a_{2}\right)$ and two charged $\left(H^{ \pm}\right) \operatorname{Higgs}$ bosons [10]. It is possible to identify $h_{1}$ or $h_{2}$ with the discovered Higgs boson $h(125)$ and obtain an additionnal low-mass scalar $h_{1}$ or pseudoscalar $a_{1}$. This light boson would preferentially have a large singlet mixture, resulting in suppressed couplings to vector bosons, preventing its discovery in classical channels so far.

Other BSM theories also motivates the search for a low-mass Higgs boson, such as dark-SUSY models [11] or general 2HDM+S [12].

In this document we review a set of searches for a light Higgs boson performed by the CMS experiment [13] with the $8 \mathrm{TeV}$ dataset. The explored mass range goes from 0.25 to $110 \mathrm{GeV}$. The $\gamma \gamma, \tau \tau, b b$ and $\mu \mu$ channels are considered, the classical Higgs boson searches channel $Z Z$ and $W W$ being forbidden kinematically. We split these searches according to two general strategies : the search for a direct production of the scalar or pseudoscalar with possibly additional objects, and the search for a pair production from the decay of a heavier Higgs boson, which could be $h(125)$. We denote a light scalar, which could be $h$ of the 2HDM or $h_{1}$ of the NMSSM, by $h$ and a light pseudoscalar, which could be $A$ of the 2HDM or $a_{1}$ of the NMSSM, by $a$. 


\section{Search for a direct production}

\section{$2.1 h \rightarrow \gamma \gamma$}

A search for a new scalar decaying into two photons is performed for a diphoton invariant mass in the range between 80 and $110 \mathrm{GeV}$ [14]. Both 2HDM and NMSSM allow the existence of such a particle with a sizeable cross-section times branching ratio in the diphoton channel, up to 3.5 times the one expected for a SM Higgs boson in the case of the NMSSM [15]. The analysis extends the method developed in CMS for the observation and the measurement of the properties of the SMlike Higgs boson discovered in 2012 [16]. It searches for a localized excess of diphoton events over a smoothly falling background due to prompt diphoton production and to events with at least one jet misidentified as a photon. Additionally, despite the application of an electron veto, a remaining contribution due to the Drell-Yan process, with the electrons misidentified as photons, needs to be added to the background model. To achieve the best sensitivity, the events are separated into four classes obtained from a multivariate classifier. The expected and observed limits on the product of the cross-section times branching ratio into two photons are presented (fig.1). No significant excess with respect to the expected limit is observed. The observed limit ranges from $75 \mathrm{fb}$ at the mass hypothesis of $80 \mathrm{GeV}$ to $42 \mathrm{fb}$ at a mass of $110 \mathrm{GeV}$. The highest excess has a significance of 1.9 $\sigma$ for a mass of $98 \mathrm{GeV}$.

$2.2 a+b b \rightarrow \tau \tau+b b$

A search for a light pseudoscalar Higgs boson decaying to a pair of $\tau$ leptons, produced in association with a $b b$ pair, is performed [17]. Pseudoscalar boson masses between 25 and $80 \mathrm{GeV}$ are probed. Three di-tau final states are considered : $\mu \tau_{h}, e \tau_{h}$ and $e \mu$. The main backgrounds are $t \bar{t}$ pairs, $W+$ jets and QCD multijets. No evidence for a pseudoscalar boson is found and upper

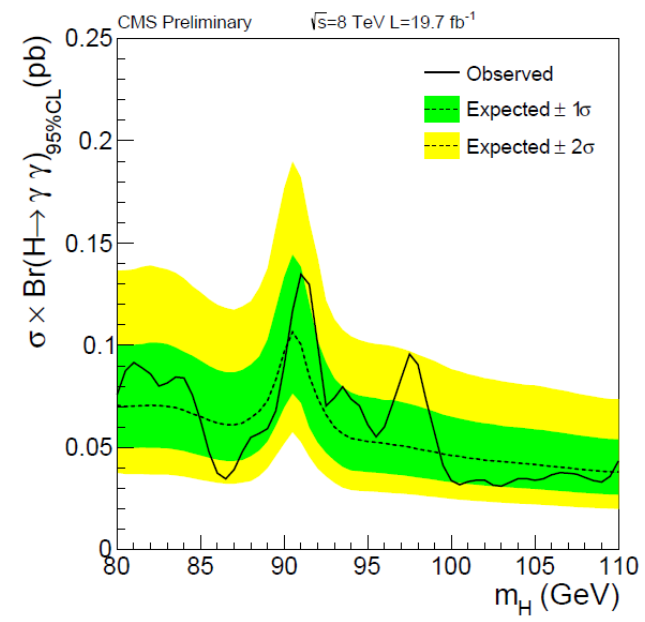

Figure 1: Expected and observed exclusion limits (95\% CL) on the product of the cross-section times branching ratio into two photons in the asymptotic CLs approximation

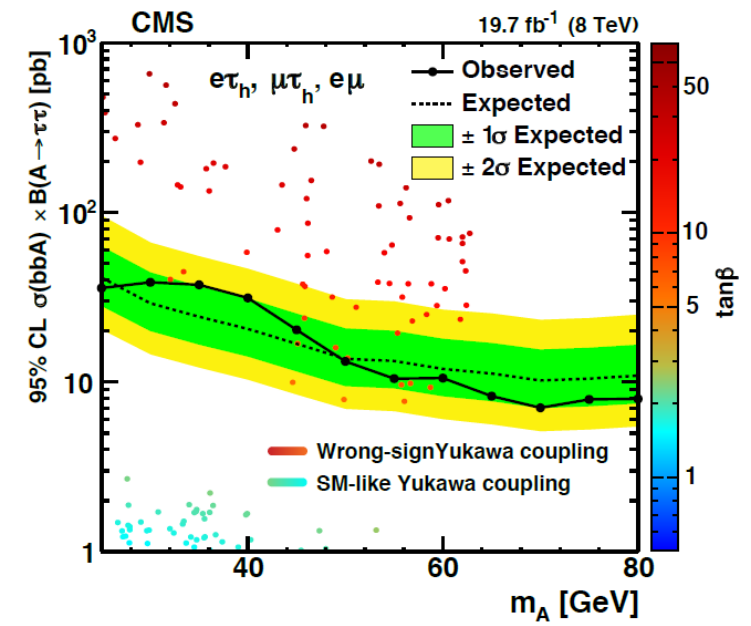

Figure 2: Expected cross sections for Type II 2HDM, superimposed on the expected and observed combined limits from the $a+b b \rightarrow \tau \tau+b b$ search 
limits are set on the production cross section times branching fraction to $\tau$ pairs between 7 and $39 \mathrm{pb}$ at the $95 \%$ confidence level (fig.2). This search excludes a pseudoscalar Higgs boson with mass below $80 \mathrm{GeV}$, in Type II 2HDM, with negative Yukawa couplings to down-type fermions (red points). It is not sensitive yet to exclude pseudoscalars with SM-like Yukawa couplings (blue points).

\section{$2.3 h+X \rightarrow b b+X$}

A search for a light scalar Higgs boson produced in supersymmetric cascades and decaying to a pair of $b$ quarks is performed [18]. The search is made in the context of the NMSSM, considering two benchmark points : the "modified P4" benchmark scenario and the "decoupled squarks" case [19] [20]. The type of process considered is the pair production of coloured supersymmetric partners, decaying to neutralinos and charginos, and subsequently into a light scalar $h_{1}$ and the lightest supersymmetric particle (LSP). Additionally a high hadronic activity is expected due to the squarks and gluinos decays to quarks. Therefore, the signature of the event is a pair of $b$ quarks coming from the decay of $h_{1}$ plus, denoted by $X$, large missing energy and at least two very energetic jets. Scalar masses between 30 and $110 \mathrm{GeV}$ are probed. The signal model is composed by a model independant $h_{1}$ resonance and a model dependant non-resonant SUSY component. No indication of a signal is observed. Limits are set on the cross section of $h_{1}$-only production times the branching fraction into $b \bar{b}$. Moreover, results are interpreted within the NMSSM benchmark scenarios. Limits are set on the NMSSM cross section times branching fraction into $b \bar{b}$ together with the prediction of the modified P4 scenario (fig.3), and limits on NMSSM parameters in the decoupled squarks scenario are obtained.

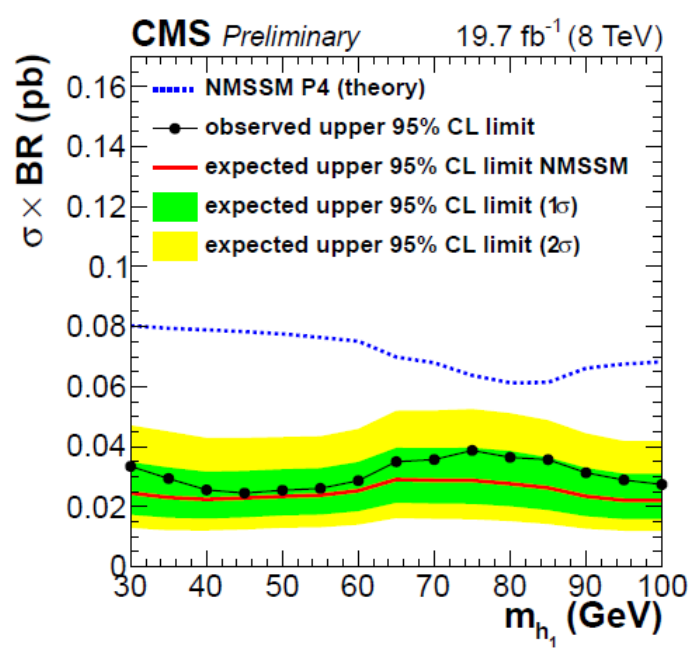

Figure 3: Upper limits for the light Higgs boson production cross section times branching fraction, for the $h+X \rightarrow b b+X$ search, in comparison to the theoretical expectations from the NMSSM P4 scenario

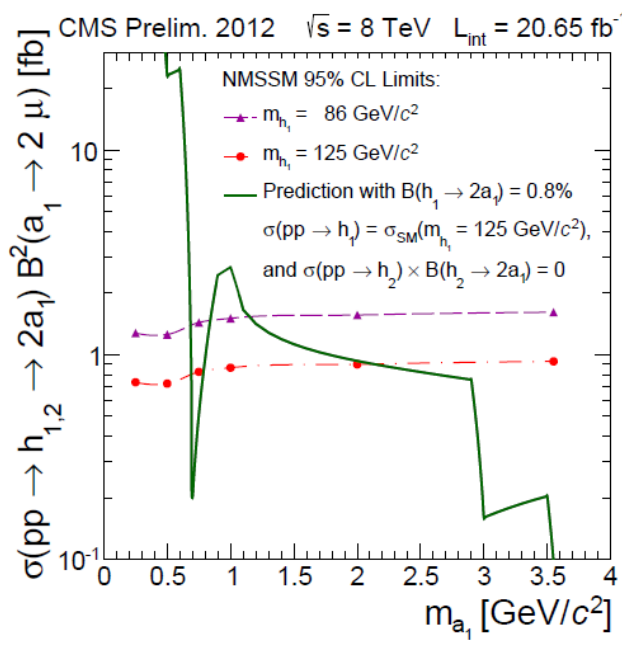

Figure 4: $95 \% \mathrm{CL}$ upper limits as functions of $m_{a_{1}}$, for the NMSSM case, on $\sigma\left(p p \rightarrow h_{1,2} \rightarrow\right.$ $2 a_{1} \times B R^{2}\left(a_{1} \rightarrow 2 \mu\right)$. The limits are compared to the predicted rate obtained using a simplified scenario from [21] 


\section{Search for a pair production}

3.1 $H \rightarrow a a / \gamma_{D} \gamma_{D} \rightarrow 4 \mu$

A search for a non-Standard-Model Higgs boson decay to a pair of new light bosons, each of which decaying into the $\mu^{+} \mu^{-}$final state, is performed [22]. Two specific scenarios are considered : the NMSSM, with muons coming from the decays of a pair of light pseudoscalars $a_{1}$, and the dark-SUSY, with muons coming from the decays of a pair of dark photons $\gamma_{D}$. The expected signature is two boosted pairs of oppositely charged muons isolated from the rest of the event activity. The anaysis strategy is to search for an excess of events in the signal region, defined as a the 2D dimuon pairs space $\left(m_{\mu \mu 1}, m_{\mu \mu 2}\right)$, with $m_{\mu \mu 1}$ close to $m_{\mu \mu 2}$ and $0.25<m_{\mu \mu}<3.55 \mathrm{GeV}$. No excess of events is observed, and a model-independent upper limit on the product of the cross section times branching fraction times acceptance is derived. Results are then interpreted in terms of the NMSSM (fig.4) and dark-SUSY scenarios.

3.2 $h(125) \rightarrow a a / h h \rightarrow 4 \tau$

Searches for non-standard decays of a Standard Model-like Higgs boson to a pair of light scalar or pseudoscalar bosons, each of which decaying into a highly boosted pair of $\tau$, are performed [23] [24]. Two complementary analyzes are dedicated to this search. Both of them look for two well separated same-sign muons, each coming for a muonic decay of a $\tau$ in each pair, in order to reduce the background. The first analysis [23] covers a mass range for the $a / h$ boson of 4 to $8 \mathrm{GeV}$. A boosted pair of tau is reconstructed from a muon with an oppositely-charged track nearby. The second analysis [24] covers a mass range of 5 to $15 \mathrm{GeV}$. A boosted pair of tau is reconstructed from the CMS standard hadronic $\tau$ reconstruction algorithm (HPS) [25], requiring the presence of a muon in the seeding jet. Two categories are built, based on the amount of missing transverse energy (MET), in order to be sensitive not only to gluon fusion (ggh) and vector boson fusion $(\mathrm{VBF})$ production modes but also to associated production with a vector boson $(\mathrm{VH})$. Both

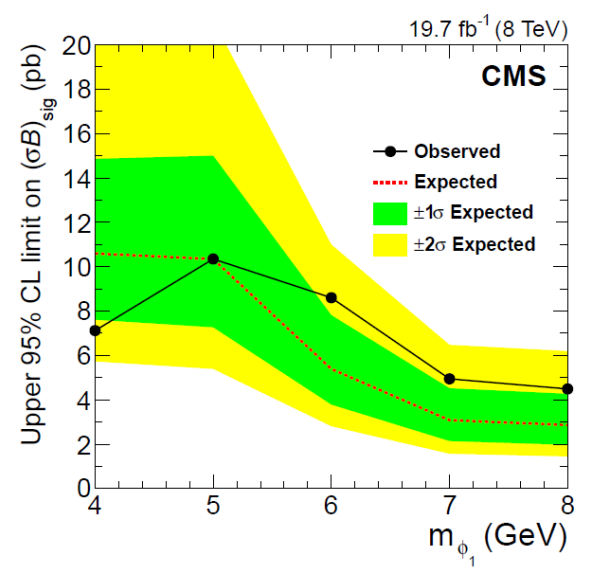

Figure 5: The observed and expected upper limits on $\sigma \times B R\left(h(125) \rightarrow \phi_{1} \phi_{1} \rightarrow 4 \tau\right)$ at $95 \% \mathrm{CL}$, as a function of the light boson mass [23]

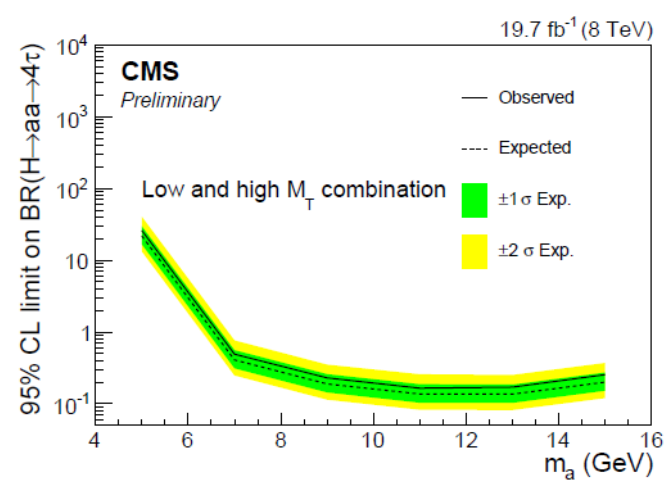

Figure 6: Expected and observed 95\% C.L. limits on the branching ratio $B R(h(125) \rightarrow a a \rightarrow 4 \tau)$. Combination of low and high MT bins [24] 
analyzes observe no excess of events, and upper limits are set on the cross section times branching fraction (fig.5) and on the branching fraction (fig.6) for the considered process.

$3.3 h(125) \rightarrow a a \rightarrow \mu \mu b b$

A search for exotic decays of a Higgs boson $h(125)$ to a pair of new light bosons $a$, where one of the light bosons decays to a pair of muons and the other one decays to a pair of $b$ quarks, is performed [26]. Both 2HDM and NMSSM predict a sizeable branching fraction for this process, up to $2.10^{-3}$ for NMSSM. The analysis covers a mass range for the pseudoscalar $a$ of 25 to $65 \mathrm{GeV}$. Only ggh production mode is considered. The sensitivity of the search to the signal is extracted using a fit to the dimuon mass distribution. No statistically significant excess of event is observed and upper limits are set on the cross section times branching ratio (fig.7) and on the branching ratio itself for the considered process.

\section{$3.4 h(125) \rightarrow a a \rightarrow \mu \mu \tau \tau$}

A search for the exotic decay of the Higgs boson $h(125)$ to a pair of light pseudoscalar bosons $a$ is performed in the final state with two muons and two taus [27]. One motivation for this search are two-Higgs-doublet models extended with a complex singlet (2HDM+S). Masses of the pseudoscalar boson between 20 and $62.5 \mathrm{GeV}$ are probed. Five di-tau decay channels are considered : ee, $\mu \tau_{h}, e \tau_{h}, e \mu$ and $\tau_{h} \tau_{h}$. The sensitivity of the search to the signal is extracted using a fit to the dimuon mass distribution. No indication of a signal is observed. Results are interpreted in the context of general $2 \mathrm{HDM}+\mathrm{S}$. First, upper limits are set on the cross section times $B R(h(125) \rightarrow a a) \times B R(a \rightarrow \tau \tau)^{2}$, using the $2 \mathrm{HDM}+\mathrm{S}$ relation between $B R(a \rightarrow \mu \mu)$ and $B R(a \rightarrow \tau \tau)$. Then, upper limits are set on the cross section, normalized to the SM, times $B R(h(125) \rightarrow a a)$ for the four types of $2 \mathrm{HDM}+\mathrm{S}$, in function of $\tan \beta$. The best sensitivity, with

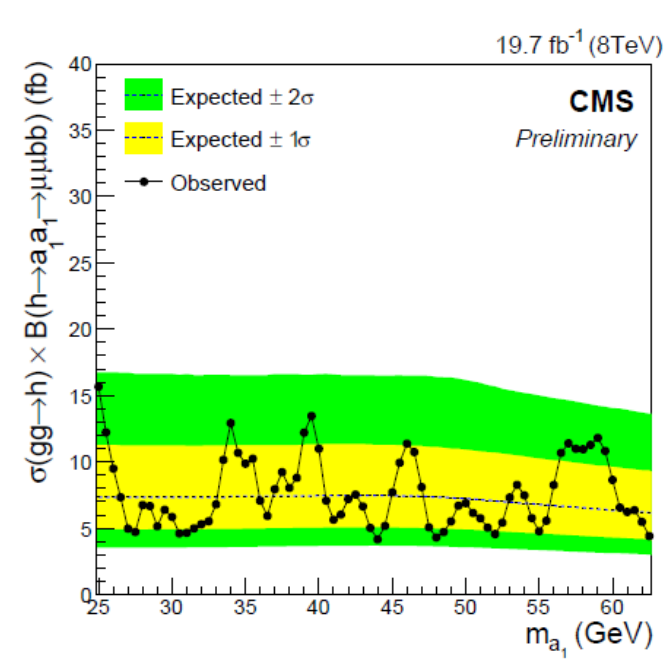

Figure 7: Observed and expected upper limits at $95 \% \mathrm{CL}$ on the Higgs boson production times $B R(h(125) \rightarrow a a \rightarrow \mu \mu b b)$

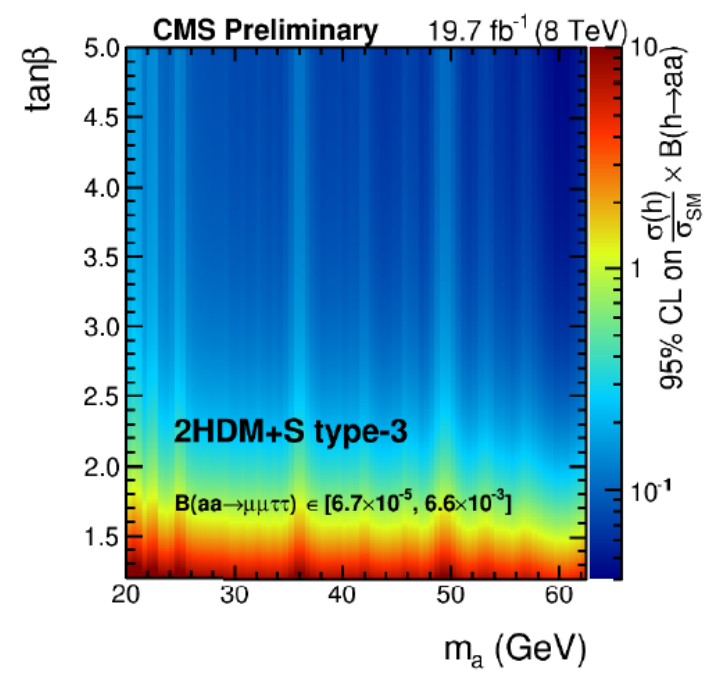

Figure 8: Observed upper limits at $95 \% \mathrm{CL}$ on $\sigma(h(125)) / \sigma_{S M} \times B R(h(125) \rightarrow a a)$ for the combination of all di-tau final states in $2 \mathrm{HDM}+\mathrm{S}$ type3 
a limit on $B R(h(125) \rightarrow a a)$ between 4 and $15 \%$, is achieved for the $2 \mathrm{HDM}+\mathrm{S}$ type III, called "lepton-specific" (fig.8).

\section{Conclusion}

Several low-mass scalar and pseudoscalar searches have been performed in CMS during run I in the range $[0.25,110] \mathrm{GeV}$. Both direct and pair production have been considered. No evidence for new particle production has been observed. The results are interpreted in terms of several BSM theories, including 2HDM and NMSSM. Wide parameter space regions of these models have been excluded and benchmark scenarios have been ruled out.

All the presented searches are being continued with run II data. Several improvements to the analyzes are foreseen, such as dedicated triggers or the consideration of new production modes and final states. In addition, the sensitivity to BSM models will beneficiate from the increase of the luminosity and the center of mass energy.

\section{References}

[1] F. Englert and R. Brout. Broken Symmetry and the Mass of Gauge Vector Mesons. Phys. Rev. Lett., 13:321-323, 1964.

[2] Peter W. Higgs. Broken Symmetries and the Masses of Gauge Bosons. Phys. Rev. Lett., 13:508-509, 1964.

[3] S. L. Glashow. Partial Symmetries of Weak Interactions. Nucl. Phys., 22:579-588, 1961.

[4] Vardan Khachatryan et al. Precise determination of the mass of the Higgs boson and tests of compatibility of its couplings with the standard model predictions using proton collisions at 7 and 8 TeV. Eur. Phys. J., C75(5):212, 2015.

[5] Georges Aad et al. Measurement of the Higgs boson mass from the $H \rightarrow \gamma \gamma$ and $H \rightarrow Z Z^{*} \rightarrow 4 \ell$ channels with the ATLAS detector using $25 \mathrm{fb}^{-1}$ of $p$ p collision data. Phys. Rev., D90(5):052004, 2014.

[6] John R. Ellis. Limits of the standard model. In PSI Zuoz. Summer School on Exploring the Limits of the Standard Model Zuoz, Engadin, Switzerland, August 18-24, 2002, 2002.

[7] G. C. Branco, P. M. Ferreira, L. Lavoura, M. N. Rebelo, Marc Sher, and Joao P. Silva. Theory and phenomenology of two-Higgs-doublet models. Phys. Rept., 516:1-102, 2012.

[8] Jeremy Bernon, John F. Gunion, Howard E. Haber, Yun Jiang, and Sabine Kraml. Scrutinizing the alignment limit in two-Higgs-doublet models. Phys. Rev., D92(7):075004, 2015.

[9] Stephen P. Martin. A Supersymmetry primer. 1997. [Adv. Ser. Direct. High Energy Phys.18,1(1998)].

[10] Ulrich Ellwanger. Higgs Bosons in the Next-to-Minimal Supersymmetric Standard Model at the LHC. Eur. Phys. J., C71:1782, 2011.

[11] Nima Arkani-Hamed, Douglas P Finkbeiner, Tracy R Slatyer, and Neal Weiner. A theory of dark matter. Physical Review D, 79(1):015014, 2009.

[12] Spencer Chang, Radovan Dermisek, John F Gunion, and Neal Weiner. Nonstandard higgs boson decays. arXiv preprint arXiv:0801.4554, 2008. 
[13] Roman Adolphi et al. The cms experiment at the cern lhc. JINST, 803:S08004-2008, 2008.

[14] CMS Collaboration. Search for new resonances in the diphoton final state in the mass range between 80 and $115 \mathrm{GeV}$ in pp collisions at $\sqrt{s}=8 \mathrm{TeV} .2015$.

[15] Jia-Wei Fan, Jun-Quan Tao, Yu-Qiao Shen, Guo-Ming Chen, He-Sheng Chen, S. Gascon-Shotkin, M. Lethuillier, L. Sgandurra, and P. Soulet. Study of diphoton decays of the lightest scalar Higgs boson in the Next-to-Minimal Supersymmetric Standard Model. Chin. Phys., C38:073101, 2014.

[16] Vardan Khachatryan et al. Observation of the diphoton decay of the Higgs boson and measurement of its properties. Eur. Phys. J., C74(10):3076, 2014.

[17] CMS Collaboration et al. Search for a low-mass pseudoscalar higgs boson produced in association with a b-bbar pair in pp collisions at sqrt $(\mathrm{s})=8$ tev. arXiv preprint arXiv:1511.03610, 2015.

[18] CMS Collaboration et al. Search for a light nmssm higgs boson produced in supersymmetric cascades and decaying into a b-quark pair. CMS PAS HIG-14-030, http://cds. cern. ch/record/2002557, 2015.

[19] Oscar Stål and Georg Weiglein. Light nmssm higgs bosons in susy cascade decays at the lhc. Journal of High Energy Physics, 2012(1):1-31, 2012.

[20] A Djouadi, M Drees, U Ellwanger, R Godbole, Cyril Hugonie, SF King, S Lehti, S Moretti, A Nikitenko, I Rottländer, et al. Benchmark scenarios for the nmssm. Journal of High Energy Physics, 2008(07):002, 2008.

[21] Radovan Dermisek and John F Gunion. New constraints on a light c p-odd higgs boson and related nmssm ideal higgs scenarios. Physical Review D, 81(7):075003, 2010.

[22] CMS collaboration et al. Search for a non-standard-model higgs boson decaying to a pair of new light bosons in four-muon final states. Physics Letters B, 726(4):564-586, 2013.

[23] CMS Collaboration et al. Search for a very light nmssm higgs boson produced in decays of the 125 gev scalar boson and decaying into tau leptons in pp collisions at sqrt $(\mathrm{s})=8$ tev. arXiv preprint arXiv:1510.06534, 2015.

[24] CMS Collaboration. Search for Higgs Decays to New Light Bosons in Boosted Tau Final States. 2015.

[25] CMS Collaboration et al. Reconstruction and identification of tau lepton decays to hadrons and tau neutrino at cms. arXiv preprint arXiv:1510.07488, 2015.

[26] CMS Collaboration. Search for exotic decays of the Higgs boson to a pair of new light bosons with two muon and two b jets in final states. 2016.

[27] CMS Collaboration. Search for the exotic decay of the Higgs boson to two light pseudoscalar bosons with two taus and two muons in the final state at $\sqrt{s}=8 \mathrm{TeV} .2016$. 